\section{'Well baby' review and screening for hepatobiliary diseases}

SIR,-We are very grateful to Dr Hall for stressing the importance of early diagnosis of biliary atresia and other cholestatic disorders of infancy ${ }^{1}$ and for drawing attention to some of the potential difficulties in achieving this effectively. ${ }^{2}$ We have always taught that all infants in whom jaundice is present beyond 14 days of age should be investigated at the earliest opportunity. Unfortunately our experience has shown that the significance of jaundice is often not recognised by either the parents or the medical attendants and this has emphasised the importance of the first formal examination for the recognition of these disorders. $^{3}$ We would be dismayed if the review were postponed until 8 weeks of age for reasons of immunisation, particularly as general immunisation rates of $95 \%$ can be achieved. ${ }^{4}$ Hence our suggestion for a four week review and for a screening programme for liver disorders.

Problems associated with the introduction of screening for cholestastic disorders along the lines we suggest should be much less than have been encountered in screening for hearing loss in the newborn. ${ }^{2}$ First of all we propose that screening should be limited to infants jaundiced at 14 days of age. ${ }^{3}$ The difficulty we do envisage may be in laboratory measurement of 'direct bilirubin' as methods of measuring this are less accurate than many other laboratory parameters. Fortunately, the infant's kidney can fractionate bilirubin better than most chemical methods. ${ }^{5}$ We contend that visual examination of the stool and urine and the use of a dipstick impregnated with diazo reagent should accurately identify infants with pathological conjugated hyperbilirubinaemia. If further confirmation is needed abnormal biochemical tests of liver function done at the same time as the direct bilirubin will be an efficient strategy for detecting hepatobiliary diseases including biliary atresia. We anticipate that the increased workload will be more than compensated for by the decreased cost coming from earlier treatment of a wide range of disorders including biliary atresia.

$$
\begin{array}{r}
\text { E R HOWARD } \\
\text { G MIELI-VERGANI } \\
\text { ALEX P MOWAT } \\
\text { Paediatric Liver Service, } \\
\text { Variety Club Children's Hospital, } \\
\text { King's College Hospital, } \\
\text { Denmark Hill, } \\
\text { London SE5 } 9 R S
\end{array}
$$

1 Hall DMB. Health for all children. Arch Dis Child 1992;67:665.

2 Hall DMB. Health for all children. 2nd Ed. Oxford: Oxford University Press, 1992:44.
3 Hussein M, Howard ER, Mieli-Vergani G, Mowat PA. Jaundice at 14 days of age: exclude biliary atresia. Arch Dis Child 1991;66:1177-9.

4 Ritchie LD, Bisset AF, Russell D, Leslie V, Thomson I. Primary and preschool immunisation in Grampian: progress and the 1990 contract. BMF 1992;304:816-9.

5 Newman GB, Hope S, Stephenson DK. Direct bilirubin measurements in jaundiced term infants. Am $\mathcal{F}$ Dis Child 1991;145:1305-9.

\section{Optimum method for growth hormone treatment}

SIR,-The paper of Stanhope et al highlights the suitability of some children for different injection devices. ${ }^{1}$ A range of products for the administration of growth hormone is available free of charge from a number of pharmaceutical companies, not just pens but also automatic injection devices.

Although the KabiPen is avaliable only in 0.5 unit increments, the point in question is whether administration of a dose on a body size or weight basis needs to be done in increments as small as 0.1 of a unit. As a large proportion of injected growth hormone is degraded subcutaneously, it is unlikely that subtle dose changes will make much difference. Further, the dose response curves for growth hormone administration suggest that altering the regimen by $0 \cdot 1$ unit increments will not make much difference to overall growth rate. Allowing a nominal dose of 20 units $/ \mathrm{m}^{2}$ to fluctuate between 19 and 21 units $/ \mathrm{m}^{2}$ body surface area per week only produces a difference in growth rate of $\pm 0.3 \mathrm{~cm} /$ year.

We are concerned by the assumption that pain experienced on injection (a subjective measure) is the major factor in achieving compliance. Our experience indicates that there are many factors influencing compliance and that pain is a surprisingly minor contributor.

S L SMITH PC HINDMARSH C G D BROOK Cobbold Laboratories, The Middlesex Hospital, Mortimer Street, London WIN $8 A A$

1 Stanhope R, Albanese A, Moyle L, Hamill G Optimum method for administration of biosynthetic human growth hormone: a randomised crossover trial of an Auto Injector and a pen injection system. Arch Dis Child 1992;67. 994-7.

Dr Stanhope comments:

Compliance with long term daily injection regimens is augmented by general conveni- ence, minimal pain, and enthusiasm for the method of administration. The recent experience of Smith and Brook of growth hormone treatment at the Middlesex Hospital has indicated that $51 \%$ of their patients were noncompliant. ${ }^{1}$ This is not only an extremely disappointing result, but also casts doubt about the validity of their growth data on response to growth hormone treatment. It was with the intention of deliberately avoiding similar results that we have focused on teaching and injection technique. We believe that the patient and/or parents should have the final choice.

1 Smith SL, Brook CGD. Evaluation of compliance to growth hormone treatment-are they getting it? Presented to the British Society for Paediatric Endocrinology, November 1991.

SIR,-It is not often a drug company wins heads and tails in a clinical trial ${ }^{1}$ so it would seem churlish to gripe, but I am moved to correct an inaccuracy of expression that $\mathrm{Dr}$ Stanhope had agreed was so at the original presentation of this work at the BPA earlier this year.

KabiPen delivers precisely the dose it is set to deliver ${ }^{2}$ and not within $+11 \%$ and $-12 \%$ as Stanhope et al report. If one wishes to deliver a dose to the precise decimal point, then KabiPen does limit one to the nearest 0.5 unit. This, I contend, is not the same as inaccurate dosing. Precise calculation of dosage is probably unnecessary (given the available flexible dosage range) and perhaps even inadvisable given the arithmetic abilities of the average British patient today. Few paediatricians prescribe other than whole or half units anyway, even when conventional syringes are used.

Finally, contrary to information given in the paper, the Auto Injector device has always been, and continues to be, available free of charge from Kabi Pharmacia.

R N WILD

Kabi Pharmacia Ltd Davy Avenue, Knowhill, Milion Keynes,
$M K 58 P H$

1 Stanhope R, Albanese A, Moyle L, Hamill G. Optimum method for administration of biosynthetic human growth hormone: a randomised crossover trial of an Auto Injector and a pen injector system. Arch Dis Child 1992;67:994-7.

2 Gluckman PD, Cutfield WS. Evaluation of a pen injection system for growth hormone treatment. Arch Dis Child 1991;66:686-8. 